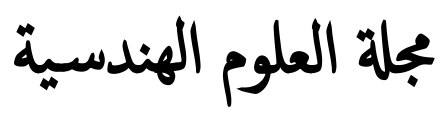

\title{
Performance Evaluation of Optical Fiber Current Transformer based Over-Current Protection
}

\author{
Sulieman Eldoud ${ }^{1, *}$, Yousif H A Rahim ${ }^{2}$ and Abdelaziz Y M Abbas ${ }^{3}$ \\ ${ }^{1}$ Lecturer, Dept. of Electrical and Electronic Engineering, Nyala University, Nyala, Sudan \\ 2 Professor, Dept. of Electrical and Computer Engineering, Karary University, Omdurman, Sudan \\ ${ }^{3}$ Assoc. Professor, Det. of Electrical and Nuclear Engineering, Sudan University of Science and Technology, Khartoum, Sudan \\ ${ }^{*}$ Corresponding author: Sulieman Eldoud (E-mail: sultona22@gmail.com )
}

Article history: Received 24 February 2019, Received in revised form 01 April 2019, Accepted 27 May 2019

\begin{abstract}
Optical Fiber Current transformers (OFCTs) are achieving increased acceptance and use in high voltage system due to their superior performance characteristics. It brings new feature to modern power system protection such as high accuracy, low weight, easy installation and no-saturation. In this paper, a new OFCT is designed and implemented a long with a digital numeric system to protect electrical-feeder from over-current due to faults. Both the optical sensing head and electronic processing block are illustrated. A detailed experimental study to confirm the performances of the device is also reported. According to the measured values of ac rms current up to $2 \mathrm{kA}$, a calibration procedure was performed. The operational characteristics of the OFCT are investigated and three different scenarios based on Prototype were carried-out to evaluate the operation of the over-current protection using OFCT. The experiential results clearly illustrate the OFCT is capable to eliminate the distortion of the electrical signals caused by conventional (CT) and hence, greatly improving the reliability of the numerical protection system.
\end{abstract}

Keywords: Current measurement; digital relay; optical fiber current transformers; optical transducers; Overcurrent protection.

\section{INTRODUCTION}

In contrast to conventional current transformers (CTs), Optical Fiber Current Transformers (OFCTs)) offer a linear response and do not suffer from saturation or hysteresis effects., Light weight, the ability to measure very large currents, and a very high dynamic range make them highly suited to power system protection applications. However, due to their optoelectronic nature, OFCT produces a very low output voltage which requires sensitive integration in order to reconstruct the measured (primary) current waveforms [1]. One of major problems of conventional current transformer (CT) is tendency to saturation due to a large magnetic field caused by high fault currents [2]. The saturation of the iron core prevents the transformer from accurately represent the primary current in the current transformer secondary, and therefore distorts current measurement [3].

The most significant advantages of OFCT are high accuracy and sensitivity, large dynamic range, fast response, immunity to electromagnetic interference and potential low cost. Also, OFCT used in explosive environments where electric sparks must be prevented because the measuring process does not involve the conduction of an electric current, it is very suitable to measure current in hazardous environments [4]. The intrinsic insulation of the optical fiber is a key 
feature for high voltage installations. It is possible to use it without any risk of discharge with the ground. In addition, OFCT has wide bandwidth making the observations of harmonics and transients easier. In addition, OFCT shows no saturation effects and can effectively replace conventional iron core transformers in specific areas with the very high currents that occur during a fault on the power system. Finally, optical fiber is small size, therefore the design of light weight and compact sensors is easy. Its installation is moreover quick, simple and can be done without interruption [5]. Among the optical current Transformers (OCTs), all-fiber current transformers are mainly based on the Faraday effect and are particularly attractive because they have better optical integration than other OCTs such as bulk-glass current transformers or space coupling OCTs [6]. The basic principal of an optical current Transformer (OCT) is based on Faraday Effect. OFCT measure the magnetic field in primary side and determine the AC current flowing in primary side based on measured magnetic field.

The Faraday Effect states that, the plane of polarization of linearly polarized light propagating in a material, which exhibits the magneto-optic effect, and which is placed in a magnetic field undergoes a rotation through an angle, which is proportional to the magnetic field component parallel to the direction of propagation.

An optical fiber current Transformer (OFCT) is developed and used in power system protection and monitoring similarly functions like conventional current transformer.

Optical current Transformers are achieving increased acceptance and usage compare to conventional CT in high voltage substations and generation plant.

This paper is focused on implementation of OFCT prototype and evaluates a performance of OFCT based over-current protection.

\section{OPERATION PRINCIPLE OF OPTICALFIBER CURRENTTRANSFORMER}

Instrument transformers are used with measuring and protective equipment in order to monitor electrical parameters such as current and voltage or to use these parameters to activate protection schemes. The general structure of an optical instrument transformer system is shown in Fig. 1. It consists of an optical source, optical fiber, sensing or modulator element, an optical detector and signal processing electronics.

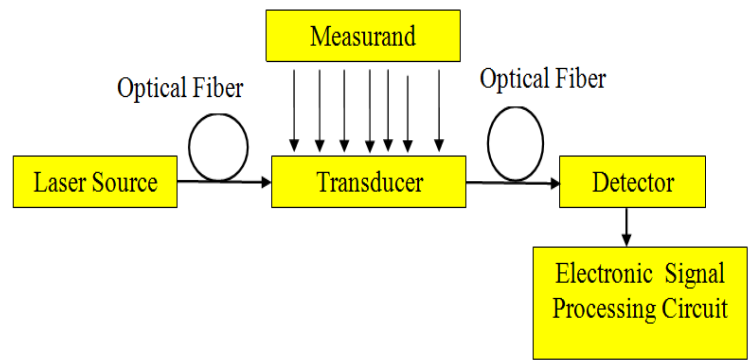

Fig. 1. Basic components of an optical fiber Instrument Transformer.

The operation of Optical fiber current transformer is based on Faraday Effect. The Faraday Effect, sometime referred to as Faraday rotation, is based on interaction between the external magnetic field and the oscillation of the electrons in the medium. The angle of rotation of linearly polarized light is proportional to the strength of the magnetic field and the cosine of the angle between the field and the propagation direction of the light wave.

According to the Faraday Effect, the rotation of the plane of polarization is proportional to the intensity of the applied magnetic field in the direction of light propagation. This principle can be used for indirect current sensing via interaction between light and a magnetic field induced by the applied current. The expression for the magnitude of Faraday rotation in radians is given by (1).

$$
\varphi=\int_{L} V \vec{B} \cdot d l
$$

where $\mathrm{V}$ is the Verdet constant, which is an optical constant that describes the strength of the Faraday and it is both dispersive and temperature dependent [7], $\mathrm{B}$ is the magnetic flux density induced by the applied current, and 1 is the length over which the magnetic field and light interact.

The Optical Current Transformers (OCTs) measures the rotation angle caused by the magnetic field and converts it into a signal of few volts proportional to the electric current. The sensitivity of OCT depends on Verdet constant which indicates the rotation angle of the polarization per unit magnetic field per unit propagation length. 
For optical fiber current transformer (OFCT), a magnetic field will be built up round the current-carrying conductor, satisfying Ampere's circuital law. Therefore, the rotation angle $\varphi_{\mathrm{F}}$ in the fiber is expressed as in (2).

$$
\varphi_{F}=V \oint H d l=N_{F} V I_{\text {elec }}
$$

Where, $N_{F}$ is the number of turns of sensing optical fiber loop, $I_{\text {elec }}$ is the electrical current in conductor.

The sensitivity of OFCT depends on Verdet constant, which indicates the rotation angle of the polarization per unit magnetic field per unit propagation length. Compared with the bulk devices, the Verdet constant of optical fibers is quite small. To overcome this problem, the optical path length can be increased by winding the fiber around a current conducting element a large number of turns [8].

\section{OPTICALFIBER CURRENTTRANSFORMER CONFIGURATION}

Several different configurations have been proposed in literature in order to detect the Faraday Effect. The detection scheme used in this work is Dual Quadrature Detection Configuration. In Dual Quadrature configuration, which is presented in Fig. 2, linearly polarized light propagates through the fiber as sensing element. While propagating through the fiber coil, the plane of polarization of the light rotates in the presence of a magnetic field. At the output end of the polarizer there is beam splitter, that separates two orthogonal polarizations in two distinct outputs, $\mathrm{S}_{1 \text { and }} \mathrm{S}_{2}$. These two signals are detected by two identical photodetectors Photodetectors output must be processed to obtained accurate electric current pass through conductor. Since, the differences normalized Sum operation $(\Delta / \Sigma)$ allows eliminating noise into output signals. Therefore, the angle of polarization rotation is reconstructed by (3).

$$
\varphi_{\mathrm{F}}\left(\mathrm{S}_{1}, \mathrm{~S}_{2}\right)=\frac{1}{2} \arcsin \left(\frac{\mathrm{S}_{1}-\mathrm{S}_{2}}{\mathrm{~S}_{1}+\mathrm{S}_{2}}\right)
$$

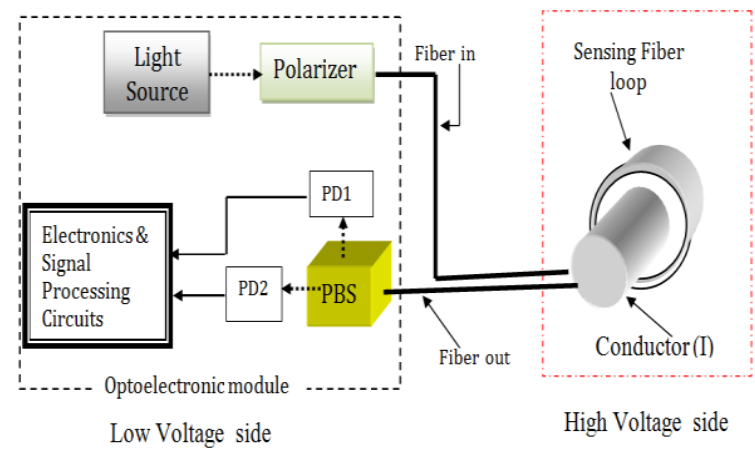

Fig. 2. Schematic of Dual Quadrature Polarimetric

\section{SYSTEM HARDWARE COMPONENTS}

A portable prototype comprises of a lightweight sensing head and a portable interrogation unit connected by fiber optic cable is tested to assess their viability as a versatile meter and protection system in high-power grids. The hardware components necessary to assemble a basic test OFCT system for measurement and protection system are described as follows:

$>$ Electrical current source (MEGGER PCITS2000/2 Primary Current Injection Test Set made by AVO-MEGGER).

DC Power Supply.

$>$ Laser source (with wavelength $632 \mathrm{~nm}$ ) He Ne laser.

Light Dependent Resistors (LDR).

Single mode optical fiber and its accessories

$>$ Optical Polarizer and objective lenses.

$>$ Polarizing Beam Splitter as optical analyzer.

PIC16F877A Microcontroller based circuit for OFCT signal processing.

PIC16F877A Microcontroller based circuit for Numerical relays implementation and signal interface circuits.

$>$ Host PC executing software.

These components are integrated together to conduct the optical fiber current transformer based power system protection test. Once all the individual circuit in the system are tested independently and proved to be working, the blocks are put together and tested. The architecture of the hardware to build complete setup used for the experimental test of the prototype is depicted in Fig. 3. In order to verify the performance of the proposed OFCT a hardware prototype has been built in the laboratory as shown in Fig. 4. 


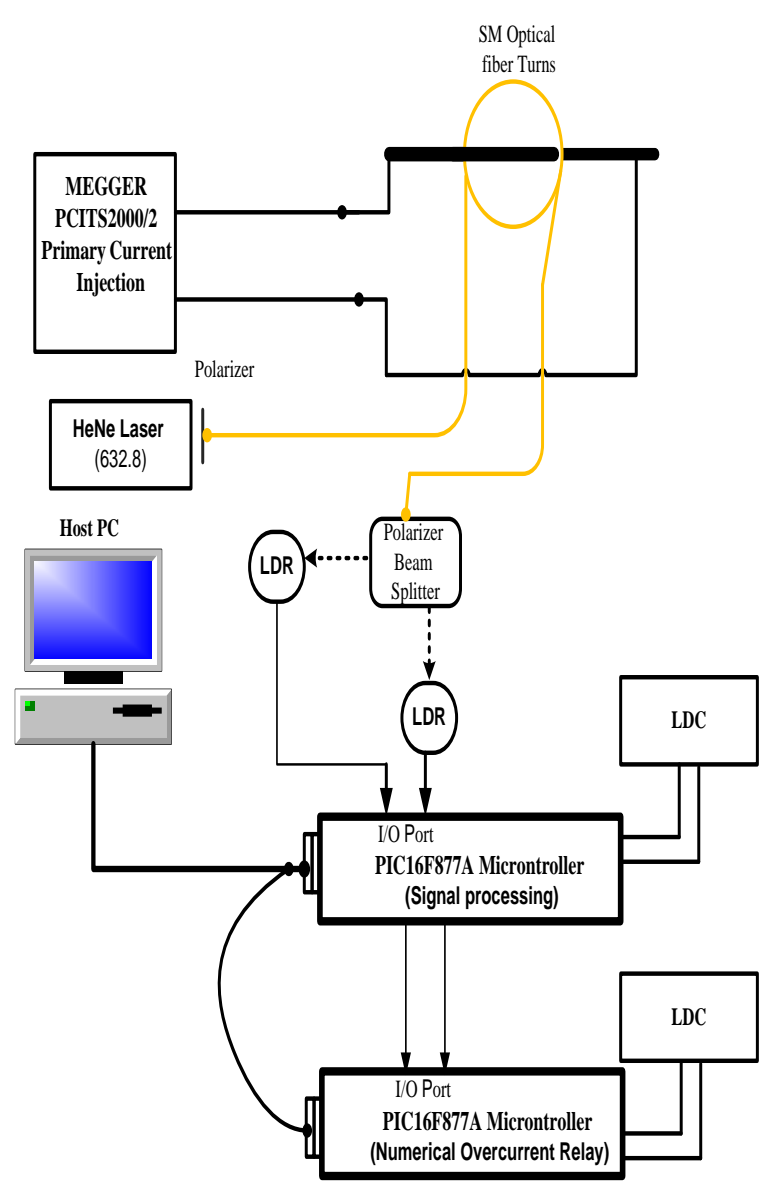

Fig. 3. Architecture of experiment for the OFCT based protection system.

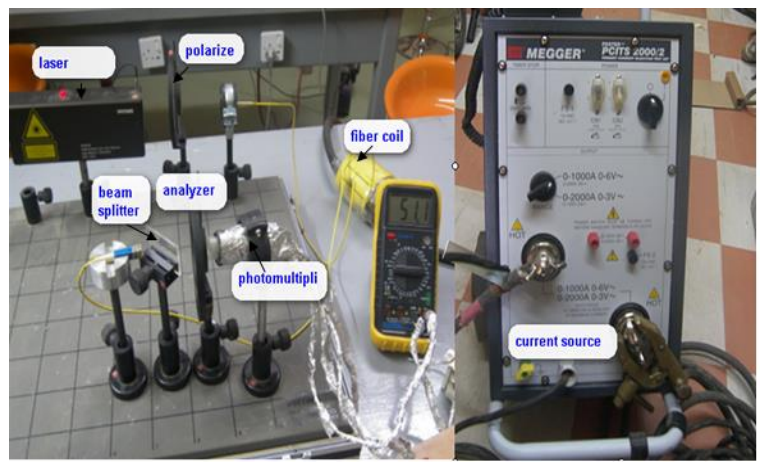

Fig. 4. Hardware prototype setup

\section{A. READOUT OF OPTICAL FIBER CURRENT TRANSFORMER CIRCUIT}

From the perspective of the electronic detection and processing circuitry, optical fiber current transformer present designer with the following challenges: Current waveform measured, Light intensity in the receiving fiber is small, and measuring harmonic contents of the current. The ultimate objective of this part is to design an electronic signal processing circuitry that uses microcontroller to read light intensities from optical part of OFCT system and automatically calculate the corresponding voltage that proportional to electrical current applied. This design is based on the PIC microcontroller as the main element in the system. The design of this system has been divided into two sections; Hardware design and Software design. A simplified scheme of the implemented electronic signal processing based PIC16F877A Microcontroller as shown in Fig. 5.

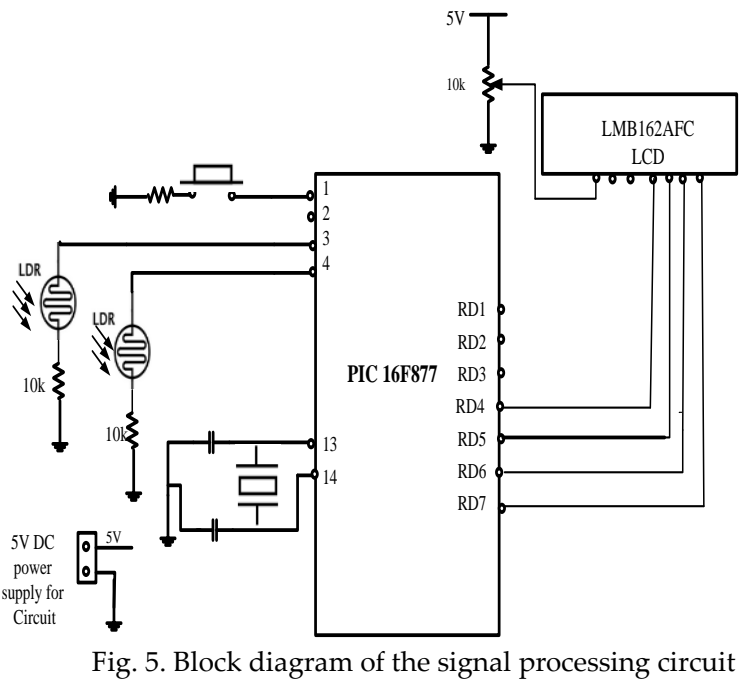

\section{B. SOFTWARE DESCRIPTION}

The mikroC® pro for PIC Compiler allows to write PIC microcontroller applications in $\mathrm{C}$ that once compiled have the efficiency and speed of assembly language. Language extensions in the mikroC Compiler give full access to all resources of the PIC microcontroller. The mikroC Compiler translates $\mathrm{C}$ source files into relocatable object modules, which contain full symbolic information for debugging with the $\mu$ Vision Debugger or an in-circuit emulator. In addition to the object file, the compiler generates a listing file, which may optionally include symbol table and cross-reference information.

The Schematic diagram of implementation of signal processing circuit using PIC16F877A microcontroller in PROTEUS® Software environment is shown in Fig. 6.

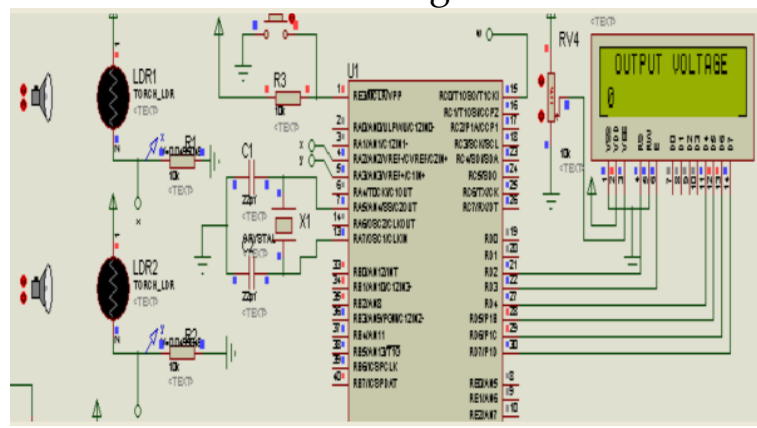

Fig. 6. Schematic diagram of implementation of signal processing circuit using PIC16F877A microcontroller 


\section{OFCT EXPERIMENTAL PROCEDURES}

The single mode optical fiber is wrapped around the current carrier about 36 turn with diameter of $8 \mathrm{~cm}$. The laser beam is coupled into optical fiber and the emerging beam after collimated micro lens is divided into two components one is transmitted and other reflected by beam splitter and each component is passed through analyzer (at angle of $0^{\circ}$ or $90^{\circ}$ ) and detected by photomultiplier in term of voltage.

The two components are detected, one directly at analyzer angle equal to $0^{\circ}$ and the other by altered output fiber terminal and changed the analyzer angle from $0^{\circ}$ to $90^{\circ}$. The polarizer angle is adjusted until two detected values are equally at no electrical current. Then the electrical current source is started and the current is increased gradually. There is no observed change in each detected values until current reached $20 \mathrm{~A}$. Then the current is increased in step of $20 \mathrm{~A}$ and each component is detected and its corresponding voltage value are displayed in LCD. In each step, the intensity of two components is detected using LDRs, which is connected to electronic signal processing circuit. The corresponding voltage value has been calculated and displayed. Fig.7 and 8 are illustrate the readout of OFCT at no electrical current path through current carrier conductor while Fig. 9 and 10 are depict the readout of OFCT at maximum electrical current.

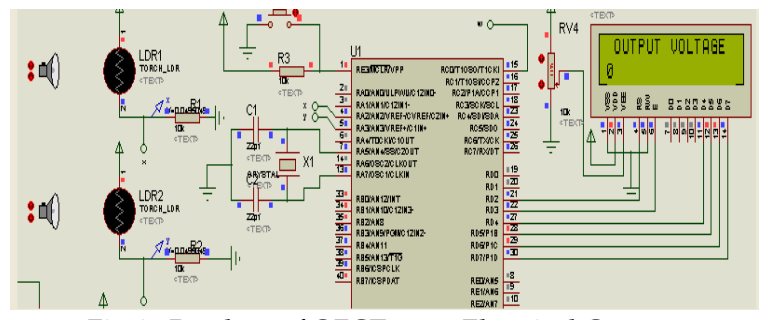

Fig. 7. Readout of OFCT at no Electrical Current

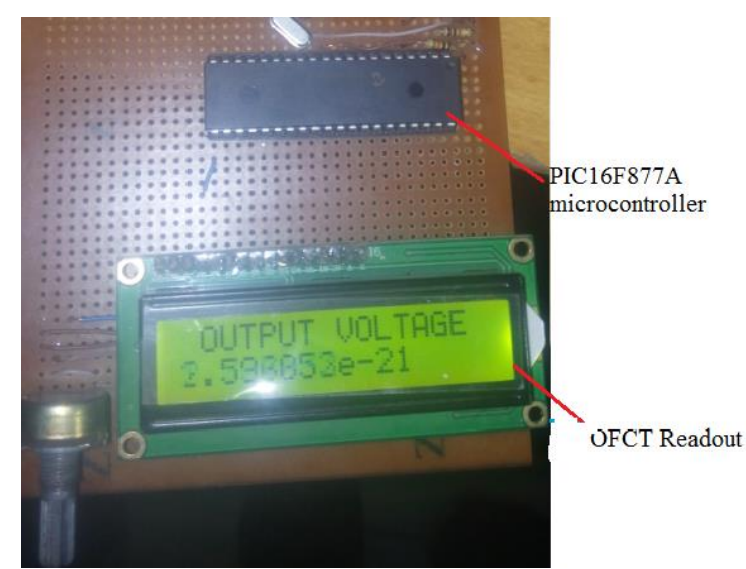

Fig. 8. Prototype Hardware Readout of OFCT at no Electrical Current

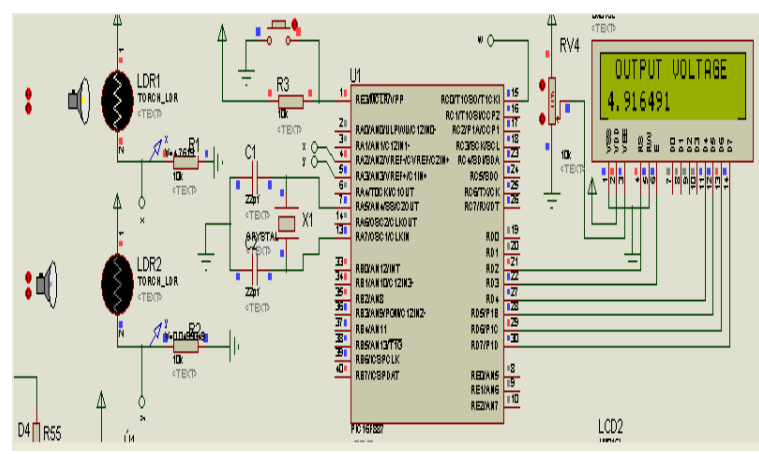

Fig. 9. Readout of OFCT corresponding Maximum Electrical Current

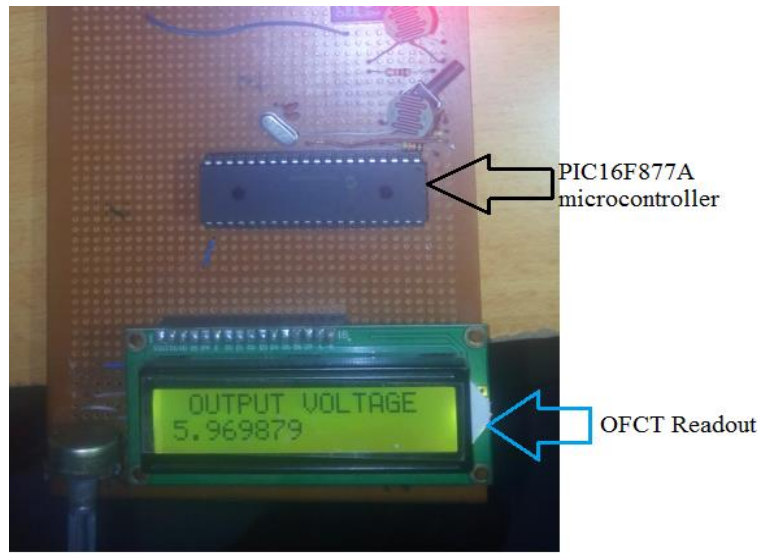

Fig. 10. Prototype Hardware Readout of OFCT corresponding Maximum Electrical Current

\section{MICROCONTROLLER BASED NUMERICAL OVERCURRENT RELAY}

Numerical Protection Relays (NPRs) are easy to operate, adjust and repair. Over current, Over/Under voltage relays are extensively used in power system protection. Implemented design is basically a System On Microcontroller [9]. Protective relays are designed in order to maintain high degree of service continuity and limit equipment damage in the power systems. The inherit advantages of microcontroller based NPRs over static NPRs with or a very limited range of applications are attractive, flexibility due to their programmable approach. Microcontroller-based NPRs can provide protection at low cost and compete with conventional relays.

Overcurrent relay (OCR) provides protection against over currents. This relay uses current inputs from an optical fiber current transformer (OFCT) and compares the measured values with preset values. If the value of input current exceeds the predetermined value, the relay detects an overcurrent and issues a trip signal to the breaker, which opens its contact to disconnect the protected equipment. When the relay detects 
a fault, the condition is called fault pickup. The relay can send a trip signal instantaneously after picking up the fault in the case of instantaneous over current relay or it can wait for a specific time before sending a trip signal in the case of time overcurrent relays. This time delay is also known as the operation time of the relay, and is computed by the relay based on the protection algorithm incorporated in PIC microcontroller as DSP. The microcontroller based overcurrent relay circuit consists of PIC16F877A, ULN2003A Darlington Transistor, Relay, LCD, LED indicator.

The overall block diagram of the proposed overcurrent protection system is shown in Fig. 11. The two different implementation methods are used to implement the overcurrent relay on the PIC16F877A microcontroller. Firstly, the overcurrent relay simulation model in Proteus software determine the value of components. The programming part is performed in mikroC®, a compiler for PIC microcontrollers. The entire program is written in embedded $\mathrm{C}$ language. Secondly, PIC16F877A microcontroller circuitry hardware is implemented and programming part for the relay is prepared in mikroC ${ }^{\circledR}$ and directly downloaded into the PIC16F877A microcontroller.

The diagram in Fig. 12 shows the Proteus schematic diagram of the Overcurrent relay.

The AC current source of $2 \mathrm{KA}$ is taken and is fed OFCT system. An LDR is used in order to change the value of the input analog quantity to the ADC port of microcontroller. From the diagram in Fig. 13 , the relay drive is connected to one of the pins of PORT B of PIC16F877A. The relay coil of $5 \mathrm{~V}$ is chosen and is connected with the apparatus that is light bulb. The light bulb is energized from a $240 \mathrm{~V}$ AC voltage source. In order to assure that the relay has tripped, for visual indication LED's are interfaced with PORT B. When the relay trips the red LED glows to show the tripping action. In order to realize the overall system, a prototype of the system is developed. The hardware setup based on PIC16F877A microcontroller and a prototype of the hardware is shown in Fig. 13.

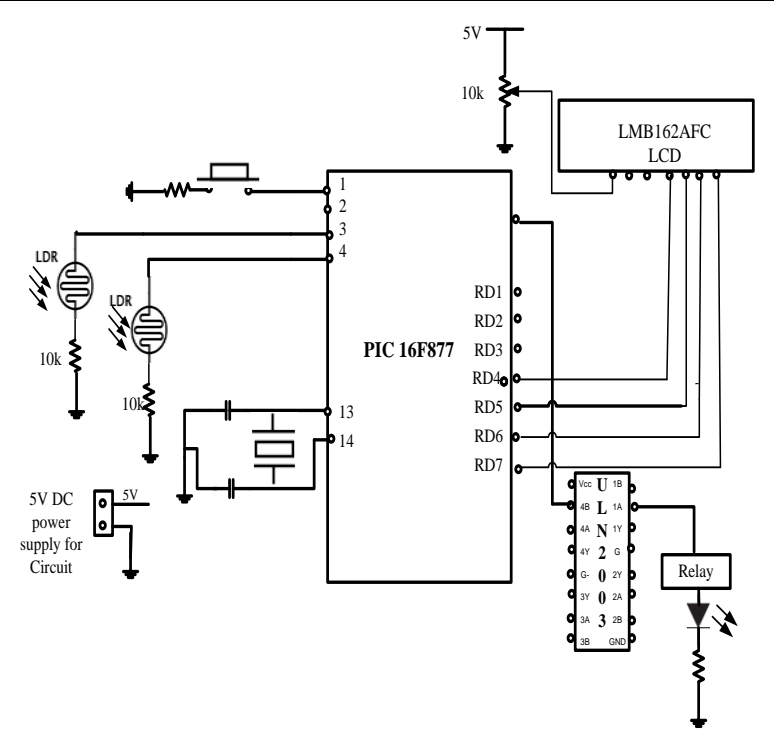

Fig. 11. PIC 16F877A based Overcurrent Relay Circuit diagram

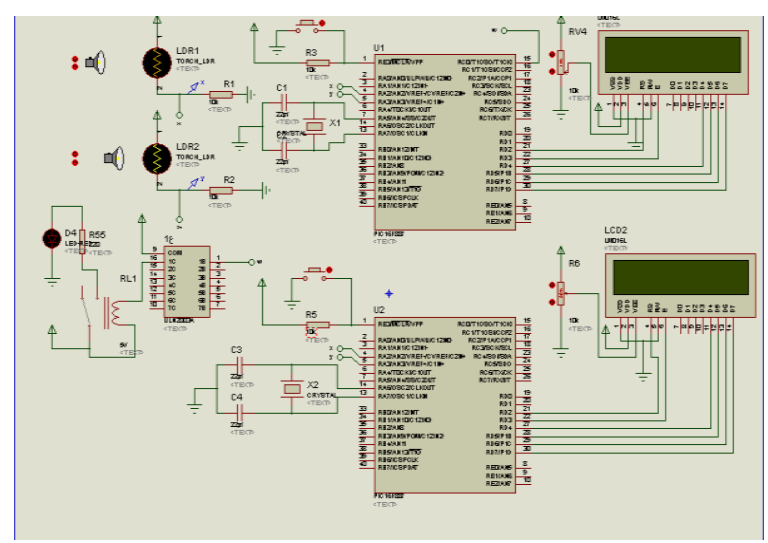

Fig. 12. Proteus schematic overcurrent relay



Fig. 13. Prototype of the Hardware System

PIC16F877A microcontroller will be used to control and operate the tripping coil in circuit breaker to measure and analyze load current from OFCT. Firstly, the load current (energizing current) will be measured using OFCT and converted from analog voltage to digital using PIC16F877A. Then the load current will be displayed on the LCD and trip circuit breaker using ULN2003A drive. 
The definite minimum time (IDMT) overcurrent relay algorithm is developed. The over current setting may be given by IDMT characteristic.

The pickup value is set in the PIC16F877A and when faults (over current) occur, PIC16F877A will energize the ULN2003A drive that will energize the circuit breaker tripping coil which will cause the circuit breaker to trip.

In order to plot the IDMT characteristics for Overcurrent relay the full load current must be known and taking into consideration 125\% overload capacity of the line thermal rating. Therefore, actual operating time is equal to relay operating time plus Circuit Bricker time. The instantaneous overcurrent relay and IDMT relay have been implemented and the LCD shows the system status and the pickup current.

For developing a prototype, the hardware is first tested with increased electrical current instead of putting a real fault at the load terminals. The circuit is simulated for normal as well as faulty operation. The pickup value is set to $2.5 \mathrm{~A}$ in the program. Using the LDRs, the analog input to the ADC port of the microcontroller is increased and once the current value exceeds, 2.5 The relay trips the apparatus from the rest of the system and as an observation the light bulb stops glowing with a red LED glowing and LCD displaying the value of pickup current value and status of relay. The normal operation of the overcurrent relay can be realized with the green LED glowing and LCD showing the peak value of line current less than setting value that. The Proteus schematic and prototype hardware are illustrated in Figs. 14 and 15, respectively. The LCD displayed the pickup current and system status, which trip has been occurred.

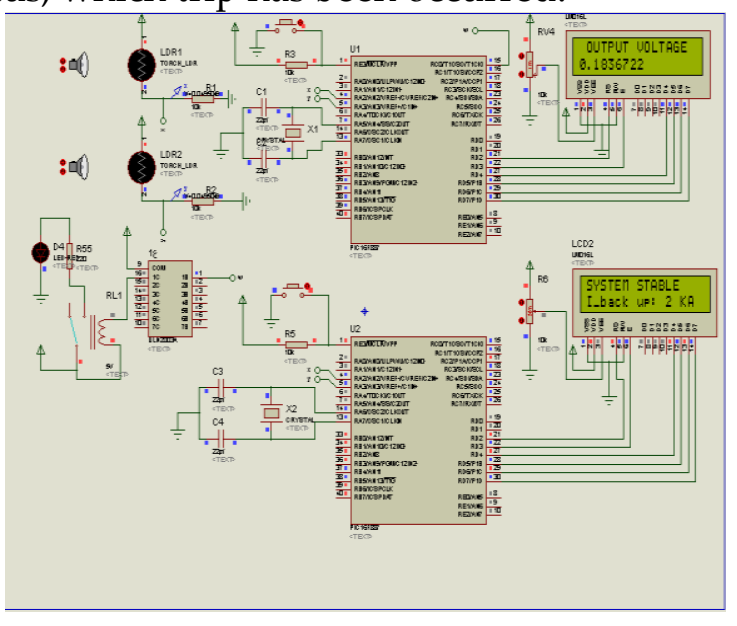

Fig. 14. Proteus schematic showing the Normal Operation.

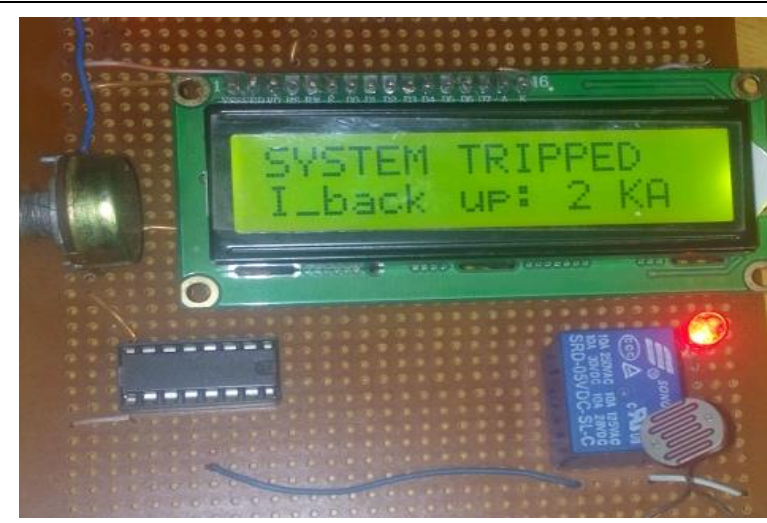

Fig. 15. Prototype Hardware Faulty Operation

The design and the realization of Microcontroller based Overcurrent are performed in Proteus and hardware prototype. The OFCT readout, system status, and operation time are summarized in Table 1 . The result carried out, there are slight variation into OFCT readout and operation time obtained from the PROTEUS simulation and hardware implementations circuit.

TABLE: I PROTOTYPE OVERCURRENT BASED OFCT RESULTS

\begin{tabular}{|c|c|c|c|}
\hline $\begin{array}{c}\text { Applied } \\
\text { Current } \\
{[\mathrm{A}]}\end{array}$ & $\begin{array}{c}\text { OFCT } \\
\text { read out } \\
{[\mathrm{V}]}\end{array}$ & $\begin{array}{c}\text { System } \\
\text { status } \\
\text { (Tripping } \\
\text { operation) }\end{array}$ & $\begin{array}{c}\text { Trip } \\
\text { Signal } \\
\text { Time } \\
\text { [Sce.] }\end{array}$ \\
\hline 50 & 0.183 & stable & -- \\
\hline 350 & 1.550 & Stable & -- \\
\hline 770 & 3.004 & Tripped & 0.123 \\
\hline 1300 & 3.635 & Tripped & 0.074 \\
\hline 2000 & 4.841 & Tripped & 0.052 \\
\hline
\end{tabular}

The experimental results carried out are satisfactory. The similar approach can also be performed in more advanced and fast microcontrollers such as Digital Signal Processors (DSP's) for both applications. It is found that this prototype can also be adopted to sense the current in the entire high voltage environment. In addition, this prototype can be used as a frequency relay by sensing the frequency of the operating voltage.

\section{CONCLUSION}

The Measuring of high electrical current in normal or faulted condition is more accurate when using OFCT without any error due to saturation effect. This is very important especially when OFCT used with protection system. Compared to conventional current transformer 
the OFCT offers a number of inherent advantages with regard to performance and ease of handling. Microcontroller based numerical relay has been implemented for the over current protection against certain abnormalities in line and is highly reactive and responds in real time. The proposed system is implemented and tested for the desired functionalities. All the calculations and decision making is carried out by a high performance eight-bit microcontroller.

\section{REFERENCES}

[1]. Das, Sukanta, Gautam Bandyopadhyay, and Prasid Syam. "Current Transformer Saturation Detection By Wavelet Transform and Compensation By Newton's Forward Interpolation." In Fifteenth National Power Systems Conference (NPSC), IIT Bombay, vol. 15, pp. 334-339. 2008.

[2]. Zhang, Z. et al, "A novel CT saturation detection algorithm for bus differential protection," 4th Int. conference on Power System Protection and Automation, 21-22 Nov-2007, New Delhi, India.
[3]. Pan,Yan, Michael Steurer, Thomas L. Baldwin, and Peter G. McLaren. "Impact of waveform distorting fault current limiters on previously installed overcurrent relays." IEEE Transactions on Power Delivery. vol. 23, no. 3, pp.1310-1318, Jul. 2008.

[4]. K. Bohnert, P. Gabus, and H. Brändle," Fiber-Optic Current and Voltage Sensors for High-Voltage Substations" 16th International Conf. on Optical Fiber Sensors, Nara, Japan, Technical Digest, pp 752-754. Oct, 2003

[5]. K. Bohnert, P. Gabus, J. Nehring, and H. Brändle, "Temperature and vibration insensitive fiber-optic current sensor," J. Lightw. Technol., vol. 20, no. 2, pp. 267-276, Feb. 2002.

[6]. Zhang, Hao, Yishen Qiu, Hui Li, Aixian Huang, Huaixi Chen, and Gaoming Li. "High-current-sensitivity all-fiber current sensor based on fiber loop architecture." Optics express 20, no. 17, pp. 18591-18599, 2012.

[7]. Z. P. Wang, Q. B. Li, and Q. Wu, "Effects of the temperature features of linear birefringence upon the sensitivity of a bulk glass current sensor," Optics \& Laser Technology. vol. 39, no. 1, pp.1 8-12, 2007.

[8]. P. Mihailovic, S. Petricevic, Z. Stojkovic, and J. Radunovic, "Development of a portable fiber optic current sensor for power systems monitoring," IEEE Trans. Instrum. Meas., vol. 53, no. 1, pp. 24-30, Feb. 2004.

[9]. M. M. Aman;, M. Q. A. Khan;, and S. A. Qazi;, "Digital Directional and Non-Directional Over Current Relays: Modelling and Performance Analysis," NED University Journal of Research, vol. 8, 2011. 